\title{
When Not to Use a Generic: Measuring HRQoL in Chronic Digestive Disease Necessitates the Use of Disease-Specific Questionnaires
}

\author{
Tiffany H. Taft ${ }^{1}$
}

Accepted: 9 December 2020 / Published online: 25 January 2021

(c) The Author(s), under exclusive licence to Springer Science+Business Media, LLC part of Springer Nature 2021

Health-Related Quality of Life (HRQoL) is a complex, subjective construct that encompasses patient perceptions of the social, emotional, occupational, functional, and financial effects of a chronic medical condition [1]. Guidance from the US Food and Drug Administration (FDA) strongly encourages measuring HRQoL as a secondary outcome in pharmaceutical clinical trials, further emphasizing the importance of questionnaire selection. In this issue of Digestive Diseases and Sciences, Chang and colleagues [2] report on how the MOS Short-Form 36 (SF-36), a widely used cross-condition measure of HRQoL, relates to symptom severity in a cohort of patients with eosinophilic esophagitis (EoE). The authors conclude the SF-36 may have limitations as a tool to evaluate $\mathrm{HRQoL}$ in these patients.

There is much debate regarding the merits of "generic" (i.e., can be applied to any condition) versus "disease-specific" (i.e., applied to only one type of condition) measures of HRQoL in terms of their application to research, especially for clinical trials [3]. In reality, since there are costs and benefits to each approach, in some instances both generic and disease-specific approaches may be appropriate. Nevertheless, in patients with EoE, a disease-specific measure may be the preferred option. A 2017 systematic review of previous measures of HRQoL in adult patients with EoE found the most widely used generic questionnaire is the SF-36, or its abbreviated version (SF-12), across six studies [4]. Strengths of the SF-36 and the SF-12 are their composite scores for physical functioning (e.g., bodily pain, mobility) and mental functioning (e.g., anxiety, social function), as well as the existence of comparator populationbased norms for healthy controls and diverse chronic illness groups. In EoE cohorts where HRQoL is assessed via the SF-36 or SF-12, the results are mixed [5]. Some studies find

Tiffany H. Taft

ttaft@northwestern.edu

1 Division of Gastroenterology and Hepatology, Northwestern University Feinberg School of Medicine, Chicago, IL, USA no differences between patients with EoE and population norms, and others identify degradations in HRQoL across physical and mental health domains [4].

An additional strength of the SF-36 is that it does not include questions about distinct disease symptoms. Though physical function is an aspect of HRQoL, the inclusion of disease symptoms within the HRQoL measurement can lower scores and distort the clinical picture of a unique and separate construct. In the present study, the authors report that both the physical and mental functioning composite scores of the SF-36 had small but significant correlations with dysphagia severity. This finding aligns with multiple studies across digestive diseases, including EoE, which consistently find relatively small-moderate relationships between SF-36 scales and symptom severity. Thus, it is safe to say that the SF-36 likely discerns between dysphagia symptoms and HRQoL in EoE. Yet, for a measure of HRQoL to be clinically useful, it also must demonstrate responsiveness and sensitivity to changes in overall health status. This is where the utility of the SF-36 in understanding EoE patient outcomes deteriorates.

One issue, replicated in the present study, is that patients with EoE inconsistently differ from population-based norms of the SF-36. In some studies, patients with EoE report significantly poorer mental function than healthy controls, whereas physical function domains are the same. Conversely, patients with EoE may report similar physical functioning to healthy controls or superior physical functioning than other disease groups (e.g., allergies, stomach diseases), yet equivalent mental health to patients with gastroesophageal reflux disease [4]. In this study's cohort, patients with EoE did not significantly differ from population-based norms. While variances are to be expected across studies, the inconsistent ability of the SF-36 to detect differences between patients with EoE and other populations is problematic.

As HRQoL is included in clinical trials of EoE, whether dietary or pharmacological, using a measure that is consistently sensitive to changes in disease status is essential. 
This is the situation where disease-specific measures may be superior to generic measures of HRQoL. When the authors compared patients with histologically active versus inactive EoE, no differences in SF-36 scores were found, concerning when taken into account with nonsignificant differences from population norms. On their own, nonsignificant differences between patients with active versus inactive disease may simply represent that disease activity is not the only contributor to a lower HRQoL in EoE. For example, the authors state that patients using empiric food elimination diets reported poorer mental functioning, with dietary treatment being the sole predictor of this outcome in regression models. Conversely, although patients using oral viscous budesonide had improved physical functioning, this treatment was not retained as a predictor in regression analyses.

A strength of the present study is its longitudinal data in a subset of 55 patients. At baseline, only $20 \%$ of patients were in histological remission. Though this percentage doubled to $44 \%$ at follow-up, HRQoL scores did not change over time across any of the SF-36 domains. It is not clear, however, what other factors may have contributed to this result, such as type of treatment or baseline anxiety or depression. Inconsistencies also exist in previously published treatment trials that use the SF-36, in spite of symptom improvement. One study reports only small improvements in HRQoL after elimination diet treatment, while another shows no change after treatment with swallowed topical steroids [4].

The authors rightfully conclude that while the strengths of the SF-36 include the ability to compare patients with EoE to both healthy controls and other chronic illness groups, it is unlikely to capture the nuances of disease-specific HRQoL in these patients. As such, disease-specific measures may be superior. Presently, there is one EoE-specific measure of HRQoL, two esophageal-specific measures, and one gastrointestinal-specific measure available. The Eosinophilic Esophagitis Quality of Life Scale for Adults (EoE-QOL-A) is a 30-item scale that evaluates specific effects of EoE on eating and diet, socialization, emotions, and anxieties about the illness and choking episodes [6]. Developed from indepth interviews with EoE patients, the EoE-QoL-A may better reflect differences in HRQoL compared with healthy controls, as well as the relationships between HRQoL and disease severity [4]. Preliminary use of the EoE-QoL-A in clinical trials shows significant differences in HRQoL improvement between patients randomized to budesonide orodispersible tablet treatment versus placebo [7], and numerical but nonsignificant improvements in HRQoL in dupilumab treatment versus placebo [8]. The EoE-QoL-A has been translated into multiple languages, including validation in a Spanish cohort.

Alternatively, the 30-item Patient Assessment of Upper Gastrointestinal Disorders-Quality of Life (PAGI-QoL) was validated in a large sample of patients with GERD, dyspepsia, and gastroparesis [9]. While not evaluated in EoE, the PAGI-QoL demonstrates high sensitivity to change over time with large effect sizes. Similarly, the Northwestern Esophageal Quality of Life Scale (NEQoL) evaluates HRQoL for all diseases affecting the esophagus, including EoE. Being shorter, with only 14 items, it facilitates comparison of EoE patients to patients with other esophageal conditions. Though the NEQoL has some overlap with EoEQoL-A domains of social functioning, emotional functioning, and eating impacts, it also includes items evaluating sleep and the financial effects of disease. Nonetheless, the NEQoL has not been widely used as yet nor evaluated for sensitivity to change. Lastly, the Gastrointestinal Quality of Life Index (GQLI) is a 36-item questionnaire that is a symptom-specific measure across both upper and lower digestive diseases, available in English and German [10]. To date, the GQLI has not been evaluated in EoE.

The present study highlights the need to use disease-specific measures of HRQoL in patients with EoE. Variability in the capacity of generic measures, such as the SF-36, to assess HRQoL in the extant literature and the existence of an EoE-specific HRQoL questionnaire should give pause to the sole use of generic measures in future EoE research. If studies wish to draw comparisons with other patient groups, the use of esophageal-specific measures is warranted. HRQoL is a complex and nuanced construct with both shared and unique domains across chronic diseases. In order to fully appreciate the effects of EoE on patient lives, as well as how potential treatments may improve or negatively affect HRQoL, disease-specific measures should be incorporated into standard research and clinical practice since their benefits outweigh those of more generic questionnaires.

\section{References}

1. Wilson IB, Cleary PD. Linking clinical variables with healthrelated quality of life: a conceptual model of patient outcomes. JAMA 1995;273:59-65

2. Chang N, Raja S, Betancourt R et al. Generic measures of quality of life are not correlated with disease activity in eosinophilic esophagitis. Dig Dis Sci. (Epub ahead of print). https://doi. org/10.1007/s10620-020-06719-z.

3. Patrick DL, Deyo RA. Generic and disease-specific measures in assessing health status and quality of life. Med Care 1989;27:S217-S232

4. Lucendo AJ, Arias-Gonzalez L, Molina-Infante J et al. Systematic review: health-related quality of life in children and adults with eosinophilic oesophagitis-instruments for measurement and determinant factors. Aliment Pharmacol Ther 2017;46:401-409

5. Brazier JE, Harper R, Jones NM et al. Validating the SF-36 health survey questionnaire: new outcome measure for primary care. BMJ 1992;305:160-164

6. Taft TH, Kern E, Kwiatek MA et al. The adult eosinophilic oesophagitis quality of life questionnaire: a new 
measure of health-related quality of life. Aliment Pharmacol Ther 2011;34:790-798

7. Straumann A, Lucendo AJ, Miehlke S et al. Budesonide orodispersible tablets maintain remission in a randomized, placebocontrolled trial of patients with eosinophilic esophagitis. Gastroenterology 2020;159:1672-1685

8. Hirano I, Dellon ES, Hamilton JD et al. Efficacy of dupilumab in a phase 2 randomized trial of adults with active eosinophilic esophagitis. Gastroenterology 2020;158:111-122 e10

9. De La Loge C, Trudeau E, Marquis P et al. Responsiveness and interpretation of a quality of life questionnaire specific to upper gastrointestinal disorders. Clin Gastroenterol Hepatol 2004:2:778-786

10. Eypasch E, Williams JI, Wood-Dauphinee S et al. Gastrointestinal Quality of Life Index: development, validation and application of a new instrument. Br J Surg 1995;82:216-222

Publisher's Note Springer Nature remains neutral with regard to jurisdictional claims in published maps and institutional affiliations. 